\title{
Editorial
}

\section{Complex Boundary Value Problems of Nonlinear Differential Equations 2014}

\author{
Xinguang Zhang, ${ }^{1}$ Yong Hong Wu, ${ }^{2}$ Dragoş-Pãtru Covei, ${ }^{3}$ and Xinan Hao ${ }^{4}$ \\ ${ }^{1}$ School of Mathematical and Informational Sciences, Yantai University, Yantai, Shandong 264005, China \\ ${ }^{2}$ Department of Mathematics and Statistics, Curtin University of Technology, Perth, WA 6845, Australia \\ ${ }^{3}$ Department of Applied Mathematics, The Bucharest University of Economic Studies, Piata Romana, 1st District, 22, \\ 010374 Bucharest, Romania \\ ${ }^{4}$ School of Mathematical Sciences, Qufu Normal University, Qufu, Shandong 273165, China
}

Correspondence should be addressed to Xinguang Zhang; zxg123242@sohu.com

Received 21 July 2014; Accepted 21 July 2014; Published 14 August 2014

Copyright (C) 2014 Xinguang Zhang et al. This is an open access article distributed under the Creative Commons Attribution License, which permits unrestricted use, distribution, and reproduction in any medium, provided the original work is properly cited.

Complex boundary value problems of nonlinear differential equations have merged as an interesting and fascinating branch of applied mathematics and pure mathematics with a wide range of applications in industry, economics, biology, physics, chemistry, social, and pure and applied sciences. The aim of this special issue is to present new approaches and theories for solving complex boundary value problems of nonlinear differential equations arising in the relative field. This special issue includes 33 high quality peer-reviewed papers which deal with different aspects of nonlinear differential equations. These papers contain some new, novel, and innovative techniques and ideas. We hope that all the papers published in this special issue can motivate and foster further scientific works and development of the research in the area of theory and applications of nonlinear differential equations.

Here, we are very grateful to all the authors and reviewers of the papers for their excellent contributions.

In the following we summarize briefly the content of the special issue.

In the paper titled "A new proof of central limit theorem for i.i.d. random variables," the authors give a new proof of central limit theorem for independent identically distributed random variables by using the viscosity solution theory of partial differential equation.

In the paper titled "Exit problems for jump processes having double-sided jumps with rational Laplace transforms," the authors consider the two-sided first exit problem for a jump process having jumps with rational Laplace transform and derive the joint distribution of the first passage time to two-sided barriers and the value of process at the first passage time. As applications, the explicit expressions of the dividend formulae for barrier strategy and threshold strategy are presented.

In the paper titled "Dynamic analysis and chaos of the $4 D$ fractional-order power system," the authors study the dynamic analysis of a fractional-order power system with parameter Q1 and firstly report about bifurcation analysis of the fractional order power system. In this work, the authors also discuss the dynamic analysis with different fractional order and different parameters and establish its numerical simulations which are provided to demonstrate the feasibility and efficacy of the analysis.

In the paper titled "Pricing of American put option under a jump diffusion process with stochastic volatility in an incomplete market," the authors study the pricing of American options in an incomplete market in which the dynamics of the underlying risky asset are driven by a jump diffusion process with stochastic volatility. By employing a risk-minimization criterion, the authors obtain the Radon-Nikodym derivative for the minimal martingale measure and consequently a linear complementarity problem for American option price. An iterative method is then established to solve the LCP 
problem for American put option price. The numerical results show that the model and numerical scheme are robust in capturing the feature of incomplete finance market, particularly the influence of market volatility on the price of American options.

In the paper titled "A two-grid finite element method for a second-order nonlinear hyperbolic equation," based on two conforming piecewise linear finite element spaces on one coarse grid with grid size $H$ and one fine grid with grid size $h$, respectively, the authors consider the two-grid finite element discretization techniques for the second-order nonlinear hyperbolic problems. With the proposed techniques, solving the nonlinear problems on the fine-grid space is reduced to solving a linear system on the fine-grid space and a nonlinear system on a much smaller space.

In the paper titled "Positive solutions for systems of nonlinear higher order differential equations with integral boundary conditions," by constructing some general type conditions and using fixed point theorem of cone, this paper investigates the existence of at least one and at least two positive solutions for systems of nonlinear higher order differential equations with integral boundary conditions. As application, some examples are given.

In the paper titled "The existence of solutions for four-point coupled boundary value problems of fractional differential equations at resonance," a four-point coupled boundary value problem of fractional differential equations is studied. Based on Mawhin's coincidence degree theory, some existence theorems are obtained in the case of resonance.

In the paper titled "Boundary stabilization of a semilinear wave equation with variable coefficients under the timevarying and nonlinear feedback," the authors investigate the boundary stabilization of a semilinear wave equation with variable coefficients under the time-varying and nonlinear feedback. By the Riemannian geometry methods, the stability results of the system under suitable assumptions of the bound of the time-varying term and the nonlinearity of the nonlinear term are obtained.

In the paper titled "Existence and uniqueness of positive solutions for a fractional switched system," the authors discuss the existence and uniqueness of positive solutions for a class of fractional switched system; the main results are based on a fixed point theorem of a sum operator and contraction mapping principle. Furthermore, two examples are also given to illustrate the results.

In the paper titled "On the study of global solutions for a nonlinear equation," the well-posedness of global strong solutions for a nonlinear partial differential equation including the Novikov equation is established provided that its initial value satisfies a sign condition and further conditions. If the mean function satisfies the sign condition, it is proved that there exists at least one global weak solution in the sense of distribution.

In the paper titled "Positive solutions for the eigenvalue problem of semipositone fractional order differential equation with multipoint boundary conditions," the author's objective is to study the existence of positive solution for the eigenvalue problem of semipositone fractional order differential equation with multipoint boundary conditions by using known Krasnosel'skii's fixed point theorem. Some sufficient conditions that guarantee the existence of at least one positive solution for eigenvalues sufficiently small and sufficiently large are established.

In the paper titled "Multiple results to some biharmonic problems," the authors study a nonlinear elliptic problem defined in a bounded domain involving biharmonic operator together with an asymptotically linear term. The results of at least three nontrivial solutions are established by using the topological degree theory and the critical groups.

In the paper titled "Stability of a class of coupled systems," the author deals with a class of coupled systems with damping terms by using multiplier method and the estimation techniques of the energy and shows that even if the kernel function is nonincreasing and integrable without additional conditions, the energy of the system decays also to zero in a good rate.

In the paper titled "The local stability of solutions for a nonlinear equation," the approach of Kruzkov's device of doubling the variables is applied to establish the local stability of strong solutions for a nonlinear partial differential equation by assuming that the initial value only lies in a suitable space.

In the paper titled "The uniqueness of solution for a class of fractional order nonlinear systems with p-Laplacian operator," the authors are concerned with the uniqueness of solutions for a class of $p$-Laplacian fractional order nonlinear systems with nonlocal boundary conditions. Based on some properties of the $p$-Laplacian operator, the criterion of uniqueness for solutions is established.

In the paper titled "Exponential stabilization of impulsive switched systems with time delays using guaranteed cost control," the authors investigate the stabilization problem for impulsive switched systems with time delays. First, exponential stability criteriaof the delayed impulsive switched systems are established by use of the Lyapunov-Krasovskii functional method. Based on these results, sufficient conditions for the existence of a guaranteed cost control are also given. Subject to these sufficient conditions, the closed-loop impulsive switched system under the guaranteed cost control law will be exponentially stable with a guaranteed cost value.

In the paper titled "Delta-nabla type maximum principles for second-order dynamic equations on time scales and applications," some delta-nabla type maximum principles for second-order dynamic equations on time scales are proved. By using these maximum principles, the uniqueness theorems of the solutions, the approximation theorems of the solutions, the existence theorem, and construction techniques of the lower and upper solutions for second-order linear and nonlinear initial value problems and boundary value problems on time scales are proved, the oscillation of second-order mixed delta-nabla differential equations is discussed, and some maximum principles for second-order mixed forward and backward difference dynamic system are proved.

In the paper titled "Some existence results of positive solution to second-order boundary value problems," the authors study the existence of positive and monotone solution to 
a class of boundary value problems by using the fixed point theorem of cone expansion and compression of functional type by Avery, Henderson, and O'Regan. Finally, four examples are provided to demonstrate the availability of the main results.

In the paper titled "Crank-Nicolson fully discrete $\mathrm{H}^{1}$ Galerkin mixed finite element approximation of one nonlinear integrodifferential model," the author considers a fully discrete $\mathrm{H}^{1}$-Galerkin mixed finite element approximation of one nonlinear integrodifferential model whichoften arises in mathematical modeling of the process of a magnetic field penetrating into a substance. By adopting the CrankNicolson discretization for time derivative, optimal order a priori error estimates for the unknown function and its gradient function are presented. A numerical example is given to verify the theoretical results.

In the paper titled "Positive solutions of a singular nonlocal fractional order differential system via Schauder's fixed point theorem," the authors establish the existence of positive solutions to a class of singular nonlocal fractional order differential system depending on two parameters. The main methods are based on Schauder's fixed point theorem.

In the paper titled "Dynamics of a predator-prey system with Beddington-DeAngelis functional response and delays," the authors consider a predator-prey system with Beddington-DeAngelis functional response and delays, in which not only the stage structure on prey but also the delay due to digestion is considered. First, a sufficient and necessary condition for the existence of a unique positive equilibrium by analyzing the corresponding locations of a hyperbolic curve and a line is given. Then, by constructing an appropriate Lyapunov function, the authors prove that the positive equilibrium is locally asymptotically stable under a sufficient condition. Finally, by using comparison theorem and the $w$-limit set theory, the global asymptotic stability of the boundary equilibrium and the positive equilibrium, a sufficient condition to assure the global asymptotic stability, respectively, are studied.

In the paper titled "Existence of positive solutions of Lotka-Volterra competition model with nonlinear boundary conditions," by using upper and lower solutions method for nonlinear boundary problems, a Lotka-Volterra competition model with nonlinear boundary conditions is considered. First, the authors investigate the existence of positive solutions in weak competition case and then prove that the system has no positive solution when one of the diffusion coefficients is sufficiently large.

In the paper titled "Characteristics weak Galerkin finite element methods for convection-dominated diffusion problems," the weak Galerkin finite element method is combined with the method of characteristics to treat the convectiondiffusion problems on the triangular mesh. The optimal order error estimates are derived for the corresponding characteristics weak Galerkin finite element procedure. Numerical tests are performed and reported.

In the paper titled "Positive solutions of a nonlinear parabolic partial differential equation," the authors deal with the existence and uniqueness of positive solutions to a class of nonlinear parabolic partial differential equations, by using some fixed point theorems for mixed monotone operators with perturbation.

In the paper titled "Optimal tracking for a divergent-type parabolic PDE system in current profile control," the authors consider the optimal tracking problem for a divergent-type parabolic PDE system, which can be used to model the spatial-temporal evolution of the magnetic diffusion process in a tokamak plasma. With the feed-forward trajectories generated by numerical optimization, a linear system to describe the error evolution is derived. The weak variation approach is then extended to cylindrical coordinates to obtain a Riccati-type PDE for feedback kernel synthesis. Then, a finite difference method is used to give numerical solutions for the Riccati-type PDE. The obtained feedback kernel is finally used to simulate the closed-loop system. The simulation results demonstrate that the proposed tracking method can attenuate the tracking error effectively.

In the paper titled "Solution of time periodic electroosmosis flow with slip boundary," an exact solution is derived for the time periodic electroosmotic flow in two-dimensional straight channels under slip boundary conditions.

In the paper titled "Solving a class of singularly perturbed partial differential equation by using the perturbation method and reproducing kernel method," the authors give the analytical solution and the series expansion solution of a class of singularly perturbed partial differential equationby combining traditional perturbation method and reproducing kernel method. The numerical example is studied to demonstrate the accuracy of the present method. Results obtained by the method indicate the method is simple and effective.

In the paper titled "Transient flows of Newtonian fluid through a rectangular microchannel with slip boundary," the authors study the transient flow of a Newtonian fluid in rectangular microchannels taking into account boundary slip. An exact solution is derived by using the separation of variables in space and Fourier series expansion in time.

In the paper titled "Hybrid intelligence model based on image features for the prediction of flotation concentrate grade," a hybrid intelligent model combining the two kinds of models is proposed in this paper. A slide window scheme is employed to update the hybrid model in order to improve its adaptability. The industrial practical data testing results show that the performance of the hybrid model is better than either of the two single models and it satisfies the accuracy and stability requirements in industrial applications.

In the paper titled "Different approximations to the solution of upper-convected Maxwell fluid over a porous stretching plate", the authors consider an incompressible magnetohydrodynamic flow of two-dimensional upper-convected Maxwell fluid over a porous stretching plate with suction and injection. The nonlinear partial differential equations are reduced to an ordinary differential equation by the similarity transformations and taking into account the boundary layer approximations. This equation is solved approximately by means of the optimal homotopy asymptotic method (OHAM).

In the paper titled "Positive solutions for impulsive differential equations with mixed monotonicity and optimal control," 
the authors consider positive solutions and optimal control problem for a second-order impulsive differential equation with mixed monotone terms. Some results on the existence of an optimal control and its stability are established. In addition, related examples are given for illustrations.

In the paper titled "Existence of positive solutions to nonlinear fractional boundary value problem with changing sign nonlinearity and advanced arguments," the authors discuss the existence of positive solutions to a class of fractional boundary value problem with changing sign nonlinearity and advanced arguments; the analysis relies on a nonlinear alternative of Leray-Schauder type. An example is given to illustrate their results.

In the paper titled "A comparative study of marketing channel multiagent Stackelberg model based on perfect rationality and fairness preference," the authors study channel consisting of a manufacturer and two retailers. As a basis for comparison, the first, multiagent Stackelberg model has been structured based on perfect rationality. Further, fairness preference theory will be embedded in marketing channel multiagent Stackelberg model, and the results show that, if the retailers have a jealous fairness preference, the manufacturer will reduce the wholesale price, retailers will increase the effort level, product sales will be increased, and the total channel utility and manufacturers' utility will be Pareto improvement, but the Pareto improvement of retailers' utility is associated with the interval of jealousy fairness preference coefficient. If the retailers have a sympathetic fairness preference, the manufacturer increases wholesale price, retailers reduce the effort level, and the total channel utility, manufacturer's utility, and retailers' utility are less than that of the no fairness preference utility.

\author{
Xinguang Zhang \\ Yong Hong Wu \\ Dragoș-Pãtru Covei
}

Xinan Hao 


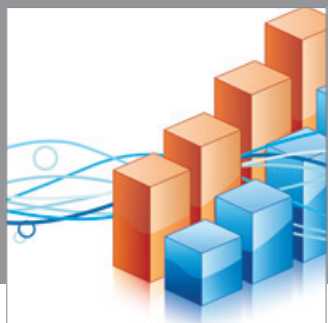

Advances in

Operations Research

mansans

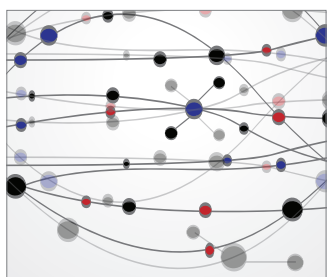

The Scientific World Journal
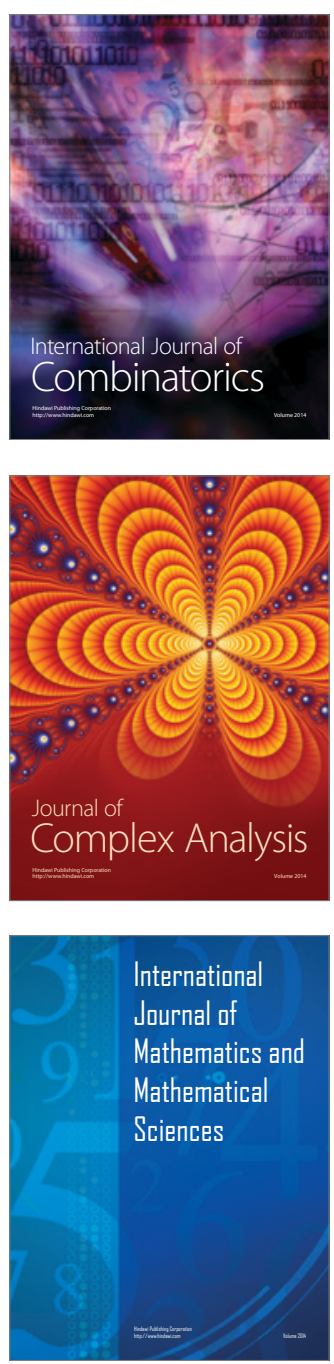
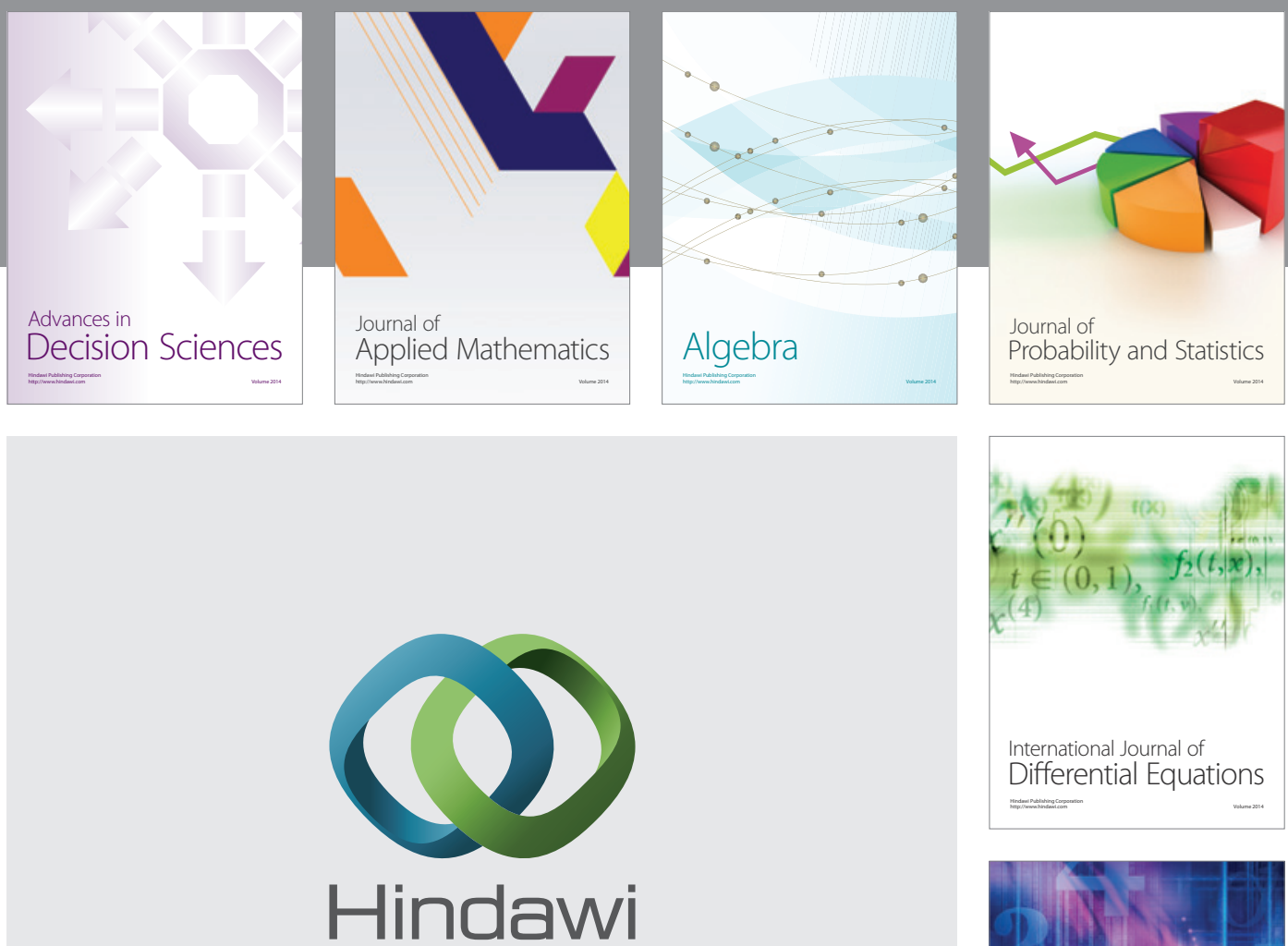

Submit your manuscripts at http://www.hindawi.com
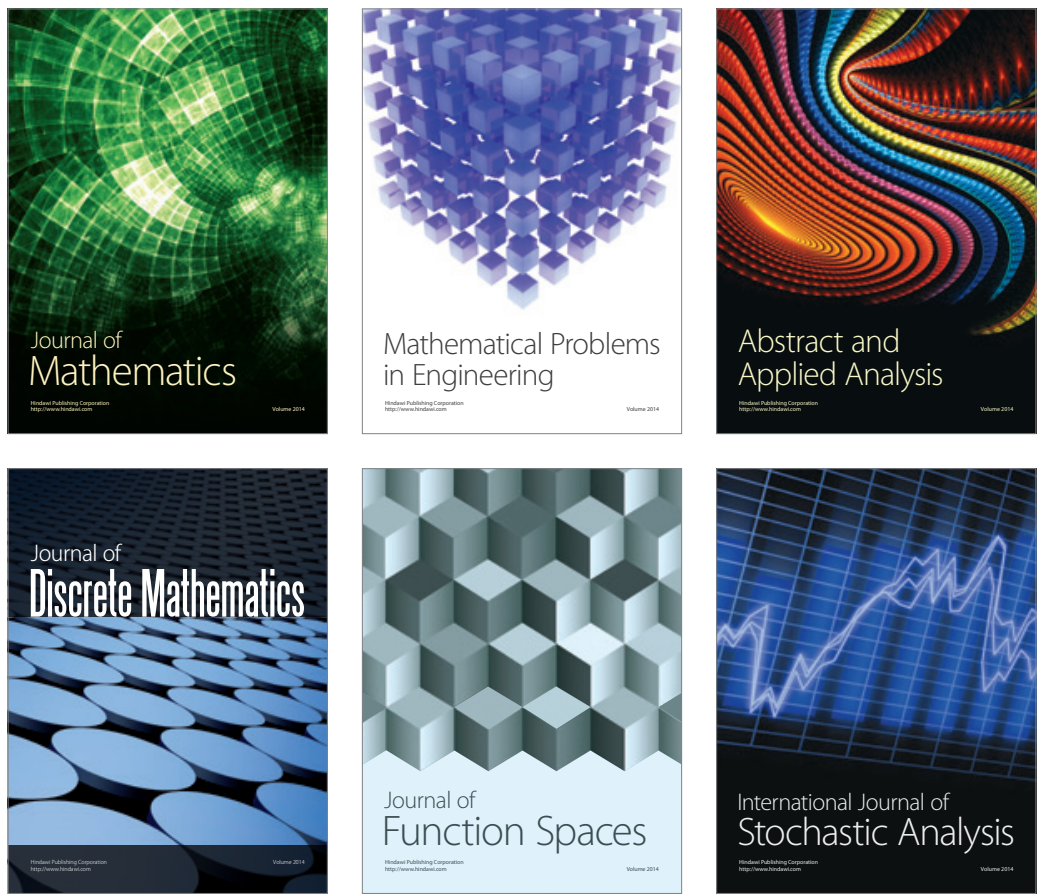

Journal of

Function Spaces

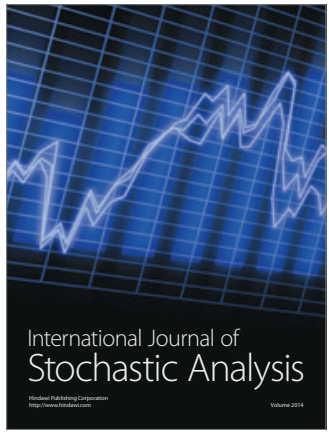

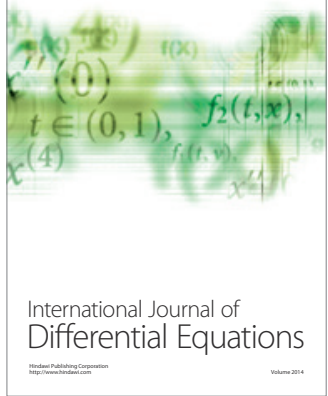
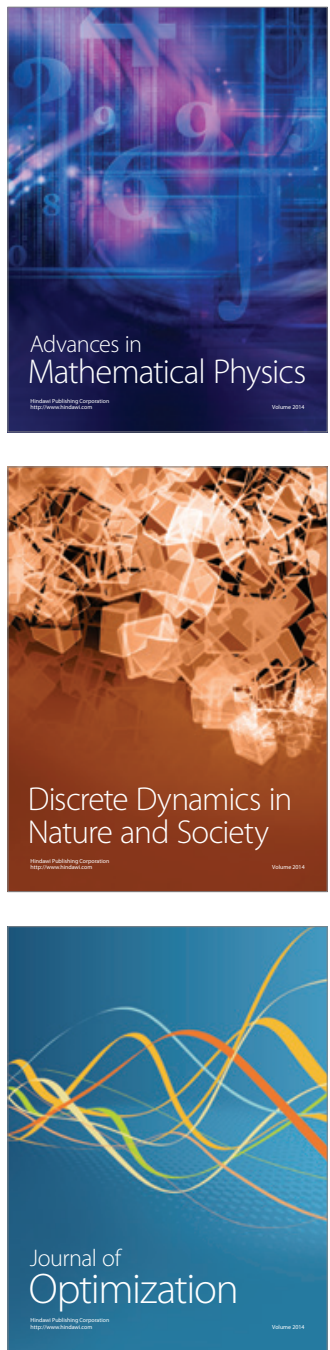